\title{
Aporte de los proyectos productivos de organismos no gubernamentales a la economía de los habitantes de Campozano
}

\section{Contribution of productive projects of non-governmental organizations to the economy of the inhabitants of Campozano}

\author{
Carlos Artemidoro Zea Barahona \\ carlos.zea@unesum.edu.ec \\ Universidad Estatal del Sur de Manabí \\ Ecuador \\ https://orcid.org/0000-0001-7546-7148 \\ Xavier Enrique Soledispa Rodríguez \\ xavier.soledispa@unesum.edu.ec \\ Universidad Estadal del Sur de Manabí \\ Ecuador \\ https://orcid.org/0000-0001-8754-9159 \\ Erick Geovanny Salazar Ponce \\ erick.salazar@unesum.edu.ec \\ Universidad Estadal del Sur de Manabí \\ Ecuador \\ https://orcid.org/0000-0002-2020-0566 \\ Luis Antonio Avilez Malave \\ reddeinvestigacionrik@fundacionkoinonia.com.ve \\ Universidad Estadal del Sur de Manabí \\ Ecuador
}

Recibido: 15 de mayo del 2019

Aprobado: 12 de junio del 2019

\section{RESUMEN}

La investigación tuvo por objetivo determinar el aporte de los proyectos productivos de organismos no gubernamentales y su relación en la economía de los habitantes de Campozano. Metodológicamente se trabajó con un tipo de investigación correlacional transeccional, la población objeto de estudio, estuvo conformada por 47 personas que han recibido financiamiento por parte de las ONG como préstamo para desarrollar actividades económicas que favorezcan el crecimiento productivo 
del sector rural ecuatoriano. Entre las principales conclusiones se tiene que los proyectos productivos han mejorado los niveles de ingresos en los habitantes de Campozano, el $100 \%$ de los encuestados indicaron que se han generado nuevas fuentes de empleo, mejores ingreso y por lo tanto que hay una mejor calidad de vida.

Descriptores: Economía agraria; Economía rural; Desarrollo agrícola; Política agraria; Política ambiental.

\begin{abstract}
The objective of the research was to determine the contribution of productive projects of non-governmental organizations and their relationship in the economy of the inhabitants of Campozano. Methodologically, a type of correlational research was carried out, the population under study was made up of 47 people who have received funding from NGOs as a loan to develop economic activities that favor the productive growth of the Ecuadorian rural sector. Among the main conclusions is that productive projects have improved income levels in the inhabitants of Campozano, $100 \%$ of respondents indicated that they have generated new sources of employment, better income and therefore that there is a better quality of lifetime.
\end{abstract}

Descriptors: Agrarian economy; Rural economy; Agricultural development; Agrarian policy, environmental policy.

\title{
INTRODUCCIÓN
}

El apoyo que se brinda a los proyectos productivos en el sector rural son muy fundamentales para el desarrollo económico alternativo por cuanto estos fortalecen las cadenas de valor del sector productivo agropecuario como lo es el cultivo, parcelas, sostenimiento, cosecha y pos cosecha, selección, procesamiento, distribución, productos que sirven de base para reactivar otros entornos microempresariales asignando valor agregado a los mismos.

Las organizaciones no gubernamentales (ONG), por medio de sus programas ha fortalecido la creación de proyectos productivos, especialmente en aquellos sectores excluidos como el sector rural o zonas periféricas, los recursos asignados por estos organismos propician la creación de microempresas que aprovechen los recursos que existen en el medio para fortalecer la economía y la calidad de vida de sus beneficiario. 
Uno de los objetivos fundamentales de los proyectos productivos es solucionar los problemas de falta de empleo, de ingresos, de explotación de los recursos naturales que existe en cada localidad o región, para esto el impulsar el establecimiento y creación de nuevas microempresas, de este modo se puede mejorar el nivel de vida de la población de dichos contextos sociales.

Los proyectos productivos cuales quiera que sea su razón de ser para la que fue ideada, significan un aporte muy significativo para quienes forman parte de él de forma directa, por cuanto van a fortalecer sus ingresos, e indirectamente de las personas que formaran parte como empleados, y de quienes producen la materia prima, la tecnología, los insumos y todos aquellos recursos que formen parte para poner en funcionamiento el proyecto.

El desarrollo productivo, está vinculado directamente con los proyectos productivos, debido a que se identificó un problema de desarrollo en los sectores de la economía, una oportunidad desaprovechada de los diferentes recursos existentes, desorganización de la comunidad, necesidades insatisfechas que motiva la creación de proyectos vinculados al desarrollo de la producción de los recursos naturales.

Los organismos no gubernamentales (ONG) tienen como una de sus principales política fomentar el desarrollo de las comunidades del sector rural y marginal, impulsando la creación de proyectos productivos a partir de los recursos que existen en cada localidad o zona, actúan con equidad e igualdad de oportunidades y generar a partir de estas actividades nuevas fuentes de empleo e ingresos a las familias de escasos recursos económicos.

Las ONG también fomentan el desarrollo de las capacidades de los productores, e las familias, de las organizaciones, con técnicas de producción acorde al avance de la ciencia, de la tecnología y los inserta en los mercados actuales considerando el fenómeno de la globalización económica para no ser absorbidos por las grandes corporaciones mundiales.

Entre las ONG del país se encuentra Visión Mundial Ecuador, la cual es una organización humanitaria que trabaja con los niños, niñas, sus familias y comunidades apoyando el fortalecimiento del sistema de protección integral, 
fomentando proyectos productivos, así como la transformación social de la niñez, adolescencia, juventud, todo en coordinación con las leyes y políticas del Estado Ecuatoriano.

En específico Visión Mundial PDA Campozano en el año 2015 destinó un presupuesto para la ejecución de proyectos productivos de $\$ 25.000$ dólares americanos y en ese año se realizaron alrededor de 15 proyectos, entre los cuales se encuentran: Crianza de aves menores, chancho, pollos, manejo y producción de miel de abejas, entrega de semillas ciclo corto maíz y frejol gandul, capacitación y entrega de hornos para para elaboración de panes, entrega de procesadoras de balanceado, entrega de maquinarias para elaboración de productos artesanales, entre otros. En los últimos 4 años Visión Mundial PDA Campozano, ha invertido en todos los proyectos productivos alrededor de $\$ 90.000$ dólares, todos direccionados a normativas del desarrollo sostenible, cuidado ambiental y buen uso de materia prima de la zona. En este sentido se plantea la siguiente interrogante de investigación: ¿Cuál es el aporte de los proyectos productivos de organismos no gubernamentales y su relación en la economía de los habitantes de Campozano? Lo cual permite establecer el siguiente objetivo de investigación: Determinar el aporte de los proyectos productivos de organismos no gubernamentales y su relación en la economía de los habitantes de Campozano.

\section{REFERENCIAL TEÓRICO}

\section{Las Organizaciones no Gubernamentales (ONG) y el apoyo productivo}

Las ONG son organizaciones sin fines de lucro, destinas a prestar asistencia, ayuda, asesorías y financiamientos para impulsar proyectos productivos de aquellos sector más desprotegidos o poco atendidos para paliar de este modo la pobreza, la falta de empleo y de inversión que existe tanto en sector rural, como urbano, específicamente en las zonas periféricas.

Los beneficios que brindas las ONG, son aplicadas sin considerar sexo, raza, religión, ideología políticas o filosóficas, es decir sin discriminación alguna, respetando los derechos constitucionales de cada país, los derechos humanos y la 
dignidad de la población beneficiada y las diferentes políticas que se apliquen direccionadas a fortalecer el desarrollo productivo de cada localidad.

Formular quiénes son los beneficiarios de la actividad de las Organizaciones no Gubernamentales, lleva a pensar de inmediato que son los seres humanos en diversas circunstancias concretas, desde las de pobreza, hasta las de enfermedades, incluyendo, por ello, las de preservación del medio ambiente y de usos tecnológicos, porque todas esas organizaciones se basan en la solidaridad humana o en la preocupación por el progreso o por la supervivencia de la humanidad (Moreno Cornejo, 2001).

Po lo tanto, son organizaciones con una relevancia a nivel global, apoyadas por entes nacionales e internacionales, dentro de estas forman parte también las fundaciones y ambas son sin fines de lucro y son creadas de forma independiente del sistema de gobierno que posee cada país, donde su principal ayuda esta direccionada a la asistencia humanitaria y social dependiendo de los diferentes problemas o crisis que se presenten en cada Estado, procuran mejorar la calidad de vida de las personas beneficiarias.

La crisis económica de nuestro país, que no es solo propia de Ecuador, también ha contribuido para el surgimiento de nuevos agentes de desarrollo. Así, asistimos a un periodo en que la ayuda internacional se canaliza cada vez en creciente proporción hacia sectores no estatales. Esta tendencia que predominará en los años 90 hará que mucha de la ayuda internacional se enrumbe a través de organismos no gubernamentales. Algunos de éstos están vinculados con la empresa privada y otros tienen vínculos diversos, incluso con el Estado (lbídem).

Por lo general el mayor financiamiento de las ONG proviene del sector privado, en algunos casos los recursos provienen del Estado y se destinan el 100\%, y en otros de ambos sectores. Hay organizaciones que su financiamiento el $100 \%$ es privado y demuestra una independencia absoluta en su accionar. Otras ONG son financiadas por la ayuda social de las personas filántropas, mundialmente se conoce que existe un gran porcentajes de personas que apoyan económicamente a las ONG.

Las ONG están se encuentran condicionadas por actuaciones locales, nacionales, regionales e internacionales, e igualmente por personas particulares y organismos 
privados con los que tienen una relación directa. Cada uno de los elementos antes señalados forma el entorno externo de las ONG, conocidos como también como el entorno macro y el entorno micro.

Quizás uno de los aspectos más controvertido cuando se habla de las ONG es el del lugar que ocupan en la sociedad y su relación con los diversos actores. En tanto que ejecutoras de proyectos, se establece una relación con las personas beneficiarias que idealmente sería una relación horizontal y con el objetivo último de fortalecer las capacidades locales. La realidad, sin embargo, es más tozuda y generalmente presenta una relación meramente asistencial, donde.

Aunque formalmente las propuestas vienen de las personas afectadas, realmente se han definido, en el mejor de los casos, en las oficinas de la ONG correspondiente. Siendo así que la participación de las personas beneficiarias es más formal que real, las necesidades suelen definirse en función de la existencia de financiación para un determinado fin, y no de los verdaderos problemas de la población. (Revilla Blanco \& Serrano Oñate, 2002).

También se puede indicar que el entorno de las ONG, está compuesto por aquellas disposiciones políticas, sociales, culturales, ambientales, financieras y económicas que rigen dentro del país donde van a operar, en algunos países se involucran a partir del Plan Nacional de Desarrollo donde se encuentran plasmado cada una de las problemáticas de los sectores sociales y productivos del Estado

Las ONG desde su aparición hasta los momentos actuales juegan un papel muy fundamental en la cimentación de un orden global e integral más justo y equitativo. En el Ecuador antes de la Constitución del 2008 tenían una actuación que no estaba regulada debidamente, a partir de 2008 en adelante son reguladas por la Secretaria Técnica de Cooperación Internacional (SETECI), quien establece las normativas de gestión regulación y planificación de estos organismos, específicamente en el año 2011. 


\section{La participación de las ONG en el Ecuador}

En el Ecuador la Secretaría Técnica de Cooperación Internacional (SETECI), es una entidad pública desconcentrada, que posee gestión técnica, administrativa y financiera propias, se encuentra adscrita al Ministerio de Relaciones Exteriores Comercio e Integración, es la encargada de recibir la información de las ONG que deseen participar en el desarrollo económico del país.

El día martes 9 de julio del año 2013, en el cantón Paján se firmó un convenio entre Visión Mundial Ecuador (VME) y el Gobierno Autónomo Descentralizado del Cantón Paján, mismo que se realizó en las instalaciones del cabildo. El objetivo del mencionado convenio es fortalecer la intervención del PDA Campozano en el cantón. Los firmantes fueron: por parte de Visión Mundial Ecuador Ximena Mora, Directora Nacional y por parte del Municipio de Paján el Alcalde Natahel Morán Cevallos. De igual manera, el convenio contempla un compromiso de trabajo conjunto de ambas instituciones en temas de protección, participación y ejercicio de derechos de los niños, niñas, adolescentes y jóvenes del Paján (Mosquera, 2013). Igualmente el día 29 de agosto del año 2013, la directora de VME y el prefecto de Manabí se reunieron en el pleno del Consejo del Gobierno Provincial en la capital manabita y en presencia de todos los miembros de la Cooperación Provincial se firmó un convenio de cooperación interinstitucional entre ambas organizaciones, mismo que fortalecería el desarrollo productivo de la parroquia Campozano del cantón Paján.

Gracias a este acuerdo de un año de duración, la Parroquia Campozano del cantón Paján, podrá impulsar sus actividades de fomento productivo en base a la participación ciudadana, la competitividad y la planificación de desarrollo efectivo, integral y sostenible de sus habitantes. Esto gracias al financiamiento, implementación y ejecución de un huerto comunitario modelo en la cabecera parroquial y de huertos familiares en las comunidades, Fincas Integrales o SIPAS (Sistema Integral de Producción Agropecuaria Sostenible), entre otros (Mosquera, 2013). 


\section{El desarrollo económico y los proyectos productivos}

Hablar de desarrollo económico es potenciar la capacidad productiva de las personas, en especial aquella que se encuentra en extrema pobreza y vulnerabilidad, pero que dentro de su lugar habitual existen recursos que con la asistencia adecuada y el desembolso de recursos pueden crear nuevas fuentes de ingresos, transformando la materia prima en nuevos bienes y servicios.

El desarrollo económico a más de promover nuevas fuentes de empleo y reactivar el desarrollo empresarial, fortalece la parte organizativa e institucional, ya que promueve políticas incluyentes, agrupa a las comunidades por medio de los proyectos productivos que pueden ser comunitarios o de grupos familiares. Esto además permite ingresar con sus productos a los mercados inclusivos a través de cadenas de comercialización nacional e internacional, en complemento de lo planteado, Salón \& Isea Argüelles (2019), destacan que es importante promover el desarrollo económico en el sector rural por cuanto esto permite establecer beneficios no solo económicos, sino, alimentarios para la población, permitiendo erradicar progresivamente la pobreza, elevándose la calidad de vida en la medida que se promueven acciones pertinentes en favorecer el progreso integral de la sociedad.

Por otro lado, Aldana Zavala \& Colina Ysea (2019), la necesidad de establecer una economía donde se promueva la visión global de los ciudadanos, es decir, que el emprendimiento y el desarrollo económico deben focalizarse en generar una propuesta sostenible como alternativa al modelo económico que solo percibe beneficios propios sin tener en cuenta al ecosistema donde se genera la empresa, siendo esto imprescindible en una comunidad mundial donde se articula la erradicación de la pobreza como objetivo de cara al 2030, siendo pertinente trabajar cooperativamente para tal fin, desde una perspectiva donde se promuevan oportunidades a los productores y emprendedores del sector rural con la finalidad de garantizar la participación productiva en la vida social del país. 


\section{METODOLOGÍA DE LA INVESTIGACIÓN}

El tipo de investigación es correlacional porque permite medir la relación existente entre las variables objeto de estudio. En este sentido, Chávez (2004) afirma que estos "estudios correlaciónales tienen como propósito determinar el grado de relación que hay entre dos variables, detectando hasta qué punto las alteraciones de una, dependen de la otra" (p. 65). En este sentido, se procuró determinar el aporte de los proyectos productivos de organismos no gubernamentales y su relación en la economía de los habitantes de Campozano, utilizándose la correlación de Spearman, con un diseño transeccional, lo que permitió a los investigadores aplicar un instrumento tipo escala de Likert, en un momento único. La población objeto de estudio, estuvo conformada por 47 personas que han recibido financiamiento por parte de las ONG como préstamo para desarrollar actividades económicas que favorezcan el crecimiento productivo del sector rural ecuatoriano.

\section{RESULTADOS}

Los resultados se presentan en base a una correlación entre los proyectos productivos de organismos no gubernamentales y la economía de los habitantes de Campozano, evidenciándose lo siguiente:

Relación proyectos productivos y economía de los habitantes

\section{Cuadro $\mathrm{N}^{\circ}-3$}

Proyectos productivos $\begin{gathered}\text { Economía de los } \\ \text { habitantes }\end{gathered}$ de

Correlación de Pearson

Sig. (bilateral)

\begin{tabular}{rrr}
$\mathrm{N}$ & 0,365 & 0,365 \\
\hline
\end{tabular}

Fuente: Investigadores (2019)

Se calculó el coeficiente de correlación de Pearson, con el fin de conocer la relación estadística entre las variables de investigación, evidenciándose una correlación 
positiva baja $(0,365)$, esto implica que los proyectos productivos influyen positivamente sobre la economía de los habitantes de Campozano. Esto implica que los proyectos han sido desarrollados efectivamente y que permiten a las personas que lo han recibido, mejorar su economía favorablemente, destacándose la necesidad de apoyar a las ONG en la consolidación de créditos que permitan a los pequeños productores del sector rural contar con una oportunidad tangible de crecer integralmente.

\section{CONCLUSIONES}

Al evidenciar cuáles son los proyectos productivos de organismos no gubernamentales que aportan significativamente a la economía de los habitantes de Campozano, se pudo constatar que en la parroquia Campozano solamente existe una ONG, que es Visión Mundial y esta tiene 10 años funcionando en esta localidad, son algunos tipos de proyectos productivos que apoyan, financian el $75 \%$ de la inversión y el 25\% restante lo hacen los beneficiarios.

Al proceder a constatar cuál es el sector de la economía que mayor aporte ha recibido de organismos no gubernamentales para potenciar el desarrollo productivo de la parroquia Campozano, se evidenció que el sector secundario es el que más se beneficia de los recursos, que la microempresa artesanal en la que mayores inversiones ha recibido lo manifestó el $77 \%$ y el 100\% de los encuestados estuvo de acuerdo en señalar que estos sectores contribuyen a la economía de Campozano.

Los proyectos productivos han mejorado los niveles de ingresos en los habitantes de Campozano, el 100\% de los encuestados indicaron que se han generado nuevas fuentes de empleo, mejores ingreso y por lo tanto que hay una mejor calidad de vida. 


\section{REFERENCIAS CONSULTADAS}

1. Aldana Zavala, J., \& Colina Ysea, F. (2019). Marketing verde en la conformación de una ciudadanía planetaria en el ámbito educativo latinoamericano. Revista San Gregorio, O(31), 150-161. Recuperado de http://revista.sangregorio.edu.ec/index.php/REVISTASANGREGORIO/article/v iew/972/15

2. Chávez, N. (2004). Introducción a la Investigación Educativa. Venezuela: Editorial Graficas, S.A.

3. FIDA-IICA. (1995). Reformas del sector agricola y el campesinado en Mexico (Vol. IV). (Agroamerica, Ed.) San José, Costa Rica: Fondo Internacional de desarrollo Agrícola. Recuperado el 23 de Abril de 2016, de https://books.google.es/books?id=m9fnanV2Nb4C\&pg=PA357\&lpg=PA357\&d $\mathrm{q}=$ Los+proyectos+productivos+y+las+Organizaciones+no+Gubernamentales+ \%280NG\%29\&source=bl\&ots=20cq8R x6IX\&sig=eiFpwiZw9rDpcOntCPCUbl MDwHc\&hl=es\&sa=X\&ved=0ahUKEwifrNa6jffMAhWDGR4KHfVXDGIQ6AEIR $\underline{\mathrm{D}}$

4. Ligteringen, E. (2004). El debate sobre el desarrollo y el futuro de las ONG (Primera ed., Vol. IX). (E. L. Deborah Eade, Ed.) España: Intermón Oxfam Editorial. Recuperado el 22 de Abril de 2016, de https://books.google.com.ec/books?id=dxdyQynQkOMC\&pg=PA275\&dq=ong \&hl=es-

419\&sa $=X \& v e d=0 a h U K E w i 564 q 54 t X M A h V C J \times 4 K H a 95 C n Q Q 6 A E I P z A G \# v=0$ nepage $\& \mathrm{q}=0$ ng $\& \mathrm{f}=$ false

5. Morales, S. d., Vaquero Lafuente, E., \& Valle López, J. (2003). El día de Europa: presente y futuro de la Unión Europea : actas de las I Jornadas en conmemoración del Día de Europa de la Universidad Pontificia Comillas de Madrid, Madrid, 8 y 9 de mayo de 2002 (Vol. I). Madrid, España: Universidad Pontifica Comillas. Recuperado el 4 de Mayo de 2016, de https://books.google.com.ec/books?id=SixN8B4QF4C\&pg=PA68\&dq=desarrollo+econ\%C3\%B3mico+concepto\&hl=es419\&sa=X\&redir esc=y\#v=onepage\&q=desarrollo\%20econ\%C3\%B3mico\%2 0concepto\& $\mathrm{f}=\mathrm{false}$

6. Moreno Cornejo, A. (2001). Economía popular y desarrollo humano (Segunda ed.). Quito, Pichincha, Ecuador: Editorial Abya Yala. Recuperado el 22 de Abril de 2016, de https://books.google.com.ec/books?id=n7wxCNxwiAgC\&pg=PA61\&dq=microe mpresa+concepto\&hl=es419\&sa $=\mathrm{X} \&$ redir esc=y $\# \mathrm{v}=0$ nepage $\& \mathrm{q}=$ =microempresa\%20 concepto\&f=false 
7. Mosquera, S. (12 de Julio de 2013). World Visión. Recuperado el 22 de Abril de 2016, de www.wvi.org: http://www.wvi.org/es/ecuador/article/firma-deconvenio-entre-visi\%C3\%B3n-mundial-ecuador-y-gobierno-de-paj\%C3\%A1n

8. Nieto Pereira, L. (2001). Cooperación para el desarrollo y ONG: una visión crítica (Vol. CIXX). España: Los Libros de la Catarata. Recuperado el 18 de Abril de 2016, de https://books.google.com.ec/books?id=jwfFJPPQToUC\&pg=PA30\&dq=teorias +economicas+de+la+ong\&hl=es419\&sa=X\&ved=0ahUKEwjir Kk4NDMAhXCRSYKHVvCaAQ6AEIGzAA\#v=onepage \&q=teorias\%20economicas\%20de\%20la\%20on g\&f=false

9. Revilla Blanco, M., \& Serrano Oñate, M. (2002). Las ONG y la política: detalles de una relación. (M. R. Blanco, Ed.) Madrid, España: Ediciones AKAL. Recuperado el 22 de Abril de 2016, de https://books.google.com.ec/books?id=vcsysyTIFlwC\&printsec=frontcover\&dq $=$ ong\&hl=es-

419\&sa=X\&ved=0ahUKEwi564q54tXMAhVCJx4KHa95CnQQ6AElJzAC\#v=on epage \&q=ong\&f=false

10.Salón, M., \& Isea Argüelles, J. (2019). El emprendimiento agroalimentario y políticas públicas en Venezuela. Revista Arbitrada Interdisciplinaria Koinonía, 4(8), 24-53. doi:http://dx.doi.org/10.35381/r.k.v4i8.235

11.Secretaria Técnia de Cooperación Internacional. (23 de Octubre de 2015). 2. Quito, Pichincha, Ecuador: SETEl. Recuperado el 22 de Abril de 2016, de http://www.cooperacioninternacional.gob.ec/normativa-para-ong/

12.Silva Lira, I., \& Sandoval, C. (2005). Desarrollo económico local/regional y fomento productivo: la experiencia chilena. Santiago de Chile, Chile: United Nations Publications. Recuperado el 26 de Abril de 2016, de https://books.google.com.ec/books?id=t74-

ahDd800C\&pg=PA69\&dq=El+desarrollo+econ\%C3\%B3mico+y+los+proyecto s+productivos\&hl=es-

419\&sa=X\&redir esc=y\#v=onepage\&q=El\%20desarrollo\%20econ\%C3\%B3mi co\%20y\%20los\%20proyectos\%20productivos\&f=false

\section{REFERENCES CONSULTED}

1. Aldana Zavala, J., \& Colina Ysea, F. (2019). Marketing verde en la conformación de una ciudadanía planetaria en el ámbito educativo latinoamericano. Revista San Gregorio, 0(31), 150-161. Recuperado de http://revista.sangregorio.edu.ec/index.php/REVISTASANGREGORIO/article/v iew/972/15 
2. Chávez, N. (2004). Introducción a la Investigación Educativa. Venezuela: Editorial Graficas, S.A.

3. FIDA-IICA. (1995). Reformas del sector agricola y el campesinado en Mexico (Vol. IV). (Agroamerica, Ed.) San José, Costa Rica: Fondo Internacional de desarrollo Agrícola. Recuperado el 23 de Abril de 2016, de https://books.google.es/books?id=m9fnanV2Nb4C\&pg=PA357\&lpg=PA357\&d $\mathrm{q}=$ Los+proyectos+productivos+y+las+Organizaciones+no+Gubernamentales+ $\% 280 N G \% 29 \&$ source=bl\&ots=20cq8Rx6IX\&sig=eiFpwiZw9rDpcOntCPCUb| MDwHc\&hl=es\&sa=X\&ved=0ahUKEwjfrNa6jffMAhWDGR4KHfVXDGIQ6AEIR $\underline{\mathrm{D}}$

4. Ligteringen, E. (2004). El debate sobre el desarrollo y el futuro de las ONG (Primera ed., Vol. IX). (E. L. Deborah Eade, Ed.) España: Intermón Oxfam Editorial. Recuperado el 22 de Abril de 2016, de https://books.google.com.ec/books?id=dxdyQynQkOMC\&pg=PA275\&dq=ong $\underline{\text { \&hl}=\mathrm{es}-}$ 419\&sa $=X \& v e d=0 a h U K E w i 564 q 54 t X M A h V C J x 4 K H a 95 C n Q Q 6 A E I P z A G \# v=0$ nepage $\& \mathrm{q}=0$ ng $\& \mathrm{f}=$ false

5. Morales, S. d., Vaquero Lafuente, E., \& Valle López, J. (2003). El día de Europa: presente y futuro de la Unión Europea : actas de las I Jornadas en conmemoración del Día de Europa de la Universidad Pontificia Comillas de Madrid, Madrid, 8 y 9 de mayo de 2002 (Vol. I). Madrid, España: Universidad Pontifica Comillas. Recuperado el 4 de Mayo de 2016, de https://books.google.com.ec/books?id=SixN-

8B4QF4C\&pg=PA68\&dq=desarrollo+econ\%C3\%B3mico+concepto\&hl=es419\&sa=X\&redir esc=y\#v=onepage\&q=desarrollo\%20econ\%C3\%B3mico\%2 0concepto\&f=false

6. Moreno Cornejo, A. (2001). Economía popular y desarrollo humano (Segunda ed.). Quito, Pichincha, Ecuador: Editorial Abya Yala. Recuperado el 22 de Abril de 2016, de https://books.google.com.ec/books?id=n7wxCNxwiAgC\&pg=PA61\&dq=microe mpresa+concepto\&hl=es-

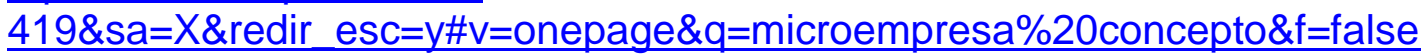

7. Mosquera, S. (12 de Julio de 2013). World Visión. Recuperado el 22 de Abril de 2016, de www.wvi.org: http://www.wvi.org/es/ecuador/article/firma-deconvenio-entre-visi\%C3\%B3n-mundial-ecuador-y-gobierno-de-paj\%C3\%A1n

8. Nieto Pereira, L. (2001). Cooperación para el desarrollo y ONG: una visión crítica (Vol. CIXX). España: Los Libros de la Catarata. Recuperado el 18 de Abril 2016, 
https://books.google.com.ec/books?id=jwfFJPPQToUC\&pg=PA30\&dq=teorias +economicas+de+la+ong\&hl=es419\&sa=X\&ved=0ahUKEwjir Kk4NDMAhXCRSYKHVvCaAQ6AEIGzAA\#v=onepage\&q=teorias\%20economicas\%20de\%20la\%20on g\&f=false

9. Revilla Blanco, M., \& Serrano Oñate, M. (2002). Las ONG y la política: detalles de una relación. (M. R. Blanco, Ed.) Madrid, España: Ediciones AKAL. Recuperado el 22 de Abril de 2016, de https://books.google.com.ec/books?id=vcsysyTIFlwC\&printsec=frontcover\&da $=$ ong\&hl=es-

419\&sa=X\&ved=0ahUKEwi564q54tXMAhVCJx4KHa95CnQQ6AEIJzAC\#v=on epage\& $q=$ ong $\&=$ false

10.Salón, M., \& Isea Argüelles, J. (2019). El emprendimiento agroalimentario y políticas públicas en Venezuela. Revista Arbitrada Interdisciplinaria Koinonía, 4(8), 24-53. doi:http://dx.doi.org/10.35381/r.k.v4i8.235

11.Secretaria Técnia de Cooperación Internacional. (23 de Octubre de 2015). 2. Quito, Pichincha, Ecuador: SETEl. Recuperado el 22 de Abril de 2016, de http://www.cooperacioninternacional.gob.ec/normativa-para-ong/

12.Silva Lira, I., \& Sandoval, C. (2005). Desarrollo económico local/regional y fomento productivo: la experiencia chilena. Santiago de Chile, Chile: United Nations Publications. Recuperado el 26 de Abril de 2016, de https://books.google.com.ec/books?id=t74ahDd800C\&pg=PA69\&dq=El+desarrollo+econ\%C3\%B3mico+y+los+proyecto s+productivos\&hl=es-

419\&sa $=X \&$ redir esc=y\#v=onepage\&q=El\%20desarrollo\%20econ\%C3\%B3mi c0\%20y\%20los\%20proyectos\%20productivos\&f=false 